\title{
A numerical experiment of backward erosion piping: kinematics and micromechanics
}

\author{
Francesco Froiio* $\quad$ Carlo Callari ${ }^{\dagger} \quad$ Andrea Francesco Rotunno ${ }^{\ddagger}$
}

(*) Univ. Lyon, École centrale de Lyon - LTDS, 69134 Ecully, France〈francesco.froiio@ec-lyon.fr

$(\dagger)$ Università del Molise - DiBT, 86100 Campobasso, Italy

( $\ddagger$ ) formerly at: Università di Roma 'Tor Vergata' - DICII, Rome, Italy

Accepted on September $13^{\text {th }} 2019$ for publication in Meccanica

\begin{abstract}
We report on a numerical experiment by which we investigated the propagation of an erosion pipe in a water saturated granular soil. The simulation was performed with a two-dimensional implementation of the coupling between the discrete element method and the lattice Boltzmann method. A synopsis of the numerical scheme is provided. The specimen and testing conditions were designed as representative of the pipe front region. The kinematics of mobilisation and fluidisation of the granular mass were investigated based on the physically-motivated definition of particle velocity thresholds. We measured a constant mass erosion rate, up to a clogging event. The study includes a micromechanical analysis of the response of the granular skeleton. We emphasise the influence of the unloading-induced damage on the erosion path, and the relevance of force chain arching as a self-organised resistance mechanism.
\end{abstract}

Keywords Granular materials · Discrete element method; Lattice Boltzmann method · Internal erosion · Backward erosion piping · Earth dams · Dikes · Levees

\section{Introduction}

Backward erosion piping is a frequent cause of failure, accidents or the degradation of service conditions for earth dams, dikes, levees and other water retaining structures [1, 2]. In research on internal erosion and the associated risk, this phenomenon is being addressed from different perspectives [3, 4]. The erosion 'pipe' forms at the downstream region of the hydraulic work and heads upstream, driven by the backward erosion mechanism. Acting as a drain, the pipe causes the progressive localisation of the seepage flow. For largeenough flow rates, particularly in the advanced stage of the process, the pipe enlarges due to tangential erosion at its wall. In the recent literature, the mechanism of pipe enlargement has been investigated or modelled with experimental, analytical and numerical tools (e.g. [5, 6, 7, 8, 9, 10]). The former mechanism of backward propagation has also been incorporated in finite element formulations at the scale of the hydraulic work 8. 9, 10, 11, 12. However, investigations specific to this erosion mechanism are still extremely rare.

This study contributes in this respect with a new numerical experiment of backward erosion. The pipe front region was modelled at the grain scale, with the granular and fluid phases being modelled via the Discrete Element (DE) and the Lattice Boltzmann (LB) methods, respectively. Compared to our previous prototype experiment 13], the simulation reported in Sect. 3 enabled a complete observation of the process of mobilisation and fluidisation of the pipe front region, due to the significantly more representative specimen and observation time. These advances were enabled by the improvement of our in-house code, including parallelisation on a shared memory architecture.

DE simulations have progressively become a common tool for investigating the behaviour of granular materials in the range of scales that commensurate with the representative grain diameter [14. The LB method stands out, among other emerging CFD tools, for the simplicity of its algorithm and the ability to model incompressible Newtonian fluids in complex flow domains. It is therefore particularly suitable as a fluid solver in DE simulations of water saturated granular materials (e.g. [7, 15, 16, 17]). The implementation of the two methods and their coupling as employed in this study are presented in the following section.

\section{$2 \quad$ Numerical model}

The implementation of the DE method was based on Ref. [18] and on state-of-the-art optimisation techniques (see [19]). A few distinctive features are presented in Sect. 2.1. with particular attention to the laws of interaction between solid particles. 
The LB method is based on the numerical resolution of the Boltzmann equation and on the related, standard concepts of statistical mechanics (e.g. the concepts of phase space and distribution function). The currently available forms of the method differ primarily by the approximation to the Boltzmann collision operator and by the discretisation of the phase space (i.e. the lattice model) [20]. The choices implemented in the aforementioned code are clarified below in Sect. 2.2. The description includes the relevant LB parameters and their relation to those of the incompressible Navies-Stokes equations.

The main features of the solid-fluid coupling scheme are introduced in Sect. 2.3. A more detailed presentation of the two numerical methods and their coupling can be found in Ref. 13.

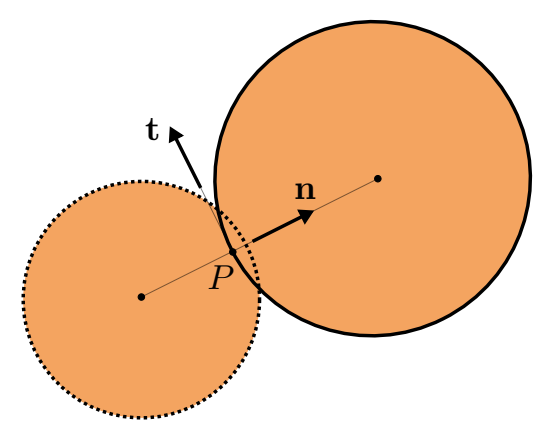

Figure 1: DE model: local basis $(\mathbf{n}, \mathbf{t})$ and nominal contact point $P$ for two interacting particles.

\subsection{Solid phase}

Grains were modelled as circular particles interacting through either frictional-viscoelastic unilateral contacts (as in [18) or brittle-viscoelastic bilateral bonds (cf. [21, 22, 23]). The latter form of interaction was introduced to model the cohesive behaviour of weakly cemented sands, or that may otherwise arise in real granular materials due to the grain interlocking. The resultant force and force moment on the generic particle were computed based on the interactions with the neighbouring particles and with the fluid (see Sect. 2.2). Gravity was neglected. The translation and rotation of particles vs. time were computed by integration of the laws of motion for rigid bodies with the velocity-Verlet scheme [24.

The law assumed for unilateral contacts can be described in terms of the local orthonormal basis and nominal contact point in Fig. 1. The unit vectors $\mathbf{n}$ and $\mathbf{t}$ set the normal and tangent contact directions, respectively. Their definitions refer to the tangent plane at contact point $P$ on the particle drawn with a continuous contour. It is assumed that the reactive force-and-couple system on such particle can be reduced to a single force with line of action through $P$. The component $F_{\mathrm{n}}$ of the force, relative to $\mathbf{n}$, is computed as

$$
F_{\mathrm{n}}=\left\{\begin{array}{cl}
-\left(k_{\mathrm{n}} \delta_{\mathrm{n}}+c_{\mathrm{n}} \dot{\delta}_{\mathrm{n}}\right) & \text { if } \delta_{\mathrm{n}}>0 \\
0 & \text { if } \delta_{\mathrm{n}} \leq 0
\end{array}\right.
$$

where $\delta_{\mathrm{n}}$ is a signed measure of the distance between the two particles, i.e. takes positive (non-positive) values in case of interpenetration (separation, resp.) of the two circular contours. Accordingly, the time rate $\dot{\delta}_{\mathrm{n}}$ is computed as $-v_{\mathrm{n}}$, with $v_{\mathrm{n}}$ the component along $\mathbf{n}$ of the velocity measured at $P$ by an observer in motion with the dotted particle. The constitutive parameters $k_{\mathrm{n}}$ and $c_{\mathrm{n}}$ are the normal contact stiffness and the normal contact dumping coefficient, respectively. The component $F_{\mathrm{t}}$ of the contact force, relative to $\mathbf{t}$, is computed as

$$
F_{\mathrm{t}}=\left\{\begin{array}{cl}
F_{\mathrm{t}}^{*} & \text { if }\left|F_{\mathrm{t}}^{*}\right|<\mu\left|F_{\mathrm{n}}\right| \\
\operatorname{sgn}\left(F_{\mathrm{t}}^{*}\right) \mu\left|F_{\mathrm{n}}\right| & \text { if }\left|F_{\mathrm{t}}^{*}\right| \geq \mu\left|F_{\mathrm{n}}\right|
\end{array}\right.
$$

in which $\mu$ is the contact friction coefficient and

$$
F_{\mathrm{t}}^{*}=-\left(k_{\mathrm{t}} \delta_{\mathrm{t}}+c_{\mathrm{t}} v_{\mathrm{t}}\right)
$$

is the candidate value. In (3), $v_{\mathrm{t}}$ is defined as $v_{\mathrm{n}}$ but referred to $\mathbf{t}, k_{\mathrm{t}}$ and $c_{\mathrm{t}}$ are constitutive parameters (cf. $k_{\mathrm{n}}$ and $c_{\mathrm{n}}$, resp. ) and the reversible tangential contact deformation, $\delta_{\mathrm{t}}$, is obtained by integration of its time rate

$$
\dot{\delta}_{\mathrm{t}}=\left\{\begin{array}{cl}
v_{\mathrm{t}} & \text { if }\left|F_{\mathrm{t}}\right|<\mu\left|F_{\mathrm{n}}\right| \\
0 & \text { if }\left|F_{\mathrm{t}}\right| \geq \mu\left|F_{\mathrm{n}}\right|
\end{array}\right.
$$

As to brittle-elastic bonds, it is assumed that

$$
F_{\mathrm{n}}=-\left(k_{\mathrm{n}} \delta_{\mathrm{n}}+c_{\mathrm{n}} \dot{\delta}_{\mathrm{n}}\right), \quad F_{\mathrm{t}}=F_{\mathrm{t}}^{*}
$$

hold unconditionally, since creation of the bond and until the tensile and shear resistance conditions

$$
F_{\mathrm{n}}>-A, \quad\left|F_{\mathrm{t}}\right|<\mu F_{\mathrm{n}}+C
$$

are satisfied, with $A$ and $C$ being the bond adhesion and bond cohesion parameters, respectively. As soon as either of $(6)$ is infringed the bond fails, i.e. is abruptly turned into a unilateral contact.

\section{$2.2 \quad$ Fluid phase}

The LB kernel of the in-house code employed in this work was based on the Bhatnagar-Gross-Krook (BGK) approximation of Boltzmann equation 25, and its common discretisation according to the D2Q9 square lattice model [26]:

$$
\begin{array}{r}
f_{\alpha}\left(\mathbf{x}+\Delta t \mathbf{c}_{\alpha}, t+\Delta t\right)=\underbrace{\underbrace{\text { BGK operator }}_{f_{\alpha}(\mathbf{x}, t)}}_{=: f_{\alpha}^{\mathrm{c}}(\mathbf{x}, t)} \\
\underbrace{}_{-\frac{\Delta t}{\tau}\left(f_{\alpha}(\mathbf{x}, t)-f_{\alpha}^{\text {eq }}(\mathbf{x}, t)\right)}
\end{array}
$$




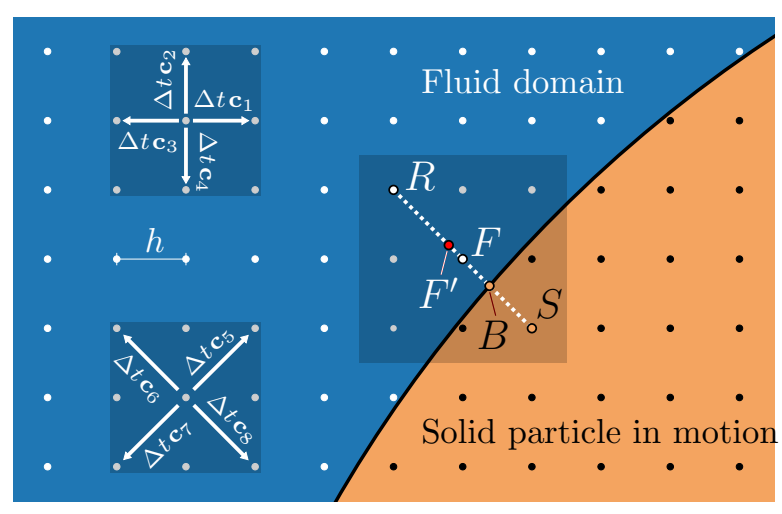

Figure 2: LB model: D2Q9 square lattice and the nodes of the interpolated bounce-back rule. Length $h$; nodeto-node displacements of a fluid particle after $\Delta t$ for the non-null velocities $\mathbf{c}_{1}$ to $\mathbf{c}_{4}$ and $\mathbf{c}_{5}$ to $\mathbf{c}_{8}$. Rear node $R$, front node $F$ and fictitious node $F^{\prime}$ of the fluid domain; node $S$ and boundary node $B$ of the solid domain.

In the above equation $\Delta t$ is the time step interval and the index $\alpha$ spans the nine allowed lattice velocities, namely the null velocity $\mathbf{c}_{0}$ and the velocities $\mathbf{c}_{1}$ to $\mathbf{c}_{8}$ in Fig. 2. The generic value $f_{\alpha}$ of the distribution function refers to fluid particles with velocity $\mathbf{c}_{\alpha}$ and to the state of the system 'just before' the instantaneous collision event at the relevant lattice position and time (e.g. $\mathbf{x}$ and $t$, resp.). The corresponding post-collision value is denoted as $f_{\alpha}^{\mathrm{c}}$. The so-called BGK operator approximates the original Boltzmann collision operator effectively for thermodynamic states of near equilibrium i.e. for $\left\{f_{\alpha}\right\}_{\alpha=0,1, \ldots, 8}$ close enough to the corresponding 9 -uplet of equilibrium values $\left\{f_{\alpha}^{\text {eq }}\right\}_{\alpha=0,1, \ldots, 8}$ computed according to the Maxwell-Boltzmann distribution. The relaxation time $\tau$ characterises the promptness by which the whole system relaxes to thermodynamic equilibrium after a small and diffuse perturbation of it (see 27]). Conversely, the so-called lattice sound speed $c_{\mathrm{s}}=h /(\sqrt{3} \Delta t)$, with $h$ as in Fig. 2 is the characteristic velocity by which the same small perturbation would propagate through the lattice if applied to a single node.

At each lattice node and time, pressure $p$ and the macroscopic fluid velocity $\mathbf{u}$ are computed through the zeroth and first order velocity moments of the distribution function:

$$
p=m c_{\mathrm{s}}^{2} \sum_{\alpha=0}^{8} f_{\alpha}, \quad \mathbf{u}=\frac{\sum_{\alpha=0}^{8} f_{\alpha} \mathbf{c}_{\alpha}}{\sum_{\alpha=0}^{8} f_{\alpha}}
$$

with $m$ the fictitious mass of the fluid particle. In the low Mach number regime, namely if the so-called computational Mach number $\mathrm{Ma}=\|\mathbf{u}\| / c_{\mathrm{S}}$ is small compared to unity, the equilibrium distribution can be computed at second order in Ma as

$$
f_{\alpha}^{\mathrm{eq}}=w_{\alpha} \frac{p}{m c_{\mathrm{s}}^{2}}\left(1+\frac{\mathbf{c}_{\alpha} \cdot \mathbf{u}}{c_{\mathrm{s}}^{2}}+\frac{\left(\mathbf{c}_{\alpha} \cdot \mathbf{u}\right)^{2}}{2 c_{\mathrm{s}}^{4}}-\frac{\|\mathbf{u}\|^{2}}{2 c_{\mathrm{s}}^{2}}\right)
$$

with

$$
\omega_{\alpha}=\left\{\begin{array}{cl}
4 / 9 & \text { if } \alpha=0 \\
1 / 9 & \text { if } \alpha=1,2,3,4 \\
1 / 36 & \text { if } \alpha=5,6,7,8
\end{array}\right.
$$

A derivation based on the Chapman-Enskog asymptotic procedure than proves that the above algorithm converges in the continuum limit to the solution of NavierStokes equations for incompressible fluids under negligible body forces 28. In particular, the momentum balance and mass balance equations are retrieved with second and first order accuracies in Ma, respectively. The asymptotic procedure identifies the relation between the parameters of the macroscopic and lattice models as

$$
\frac{\eta}{\rho_{\mathrm{f}}}=\frac{1}{3}\left(\frac{\tau}{\Delta t}-\frac{1}{2}\right) \frac{h^{2}}{\Delta t}, \quad \frac{\tau}{\Delta t}>\frac{1}{2}
$$

in which $\eta$ and $\rho_{\mathrm{f}}$ are the dynamic viscosity and mass density of the fluid, respectively.

A drawback of the attractive simplicity of the above algorithm is a certain degree of numerical instability. Solutions to this inconvenience come of course at additional numerical cost. Improved numerical stability can be obtained by a more accurate approximation of the Boltzmann collision operator, as with the Multiple Relaxation Time (MRT) approach 29] which we used in [13. Also effective is, alternatively, a substantial increase of the spatial resolution of the lattice model (i.e. reduction of $h$ ). For the numerical test of the present study we followed the latter option, whose additional computation cost was partly adsorbed by the parallelisation of the LB algorithm.

Boundary conditions of the pressure-velocity type were implemented in the form suggested in Ref. [30]. Assume in particular that the leftmost vertical alignment of nodes in Fig. 2 is a boundary of the fluid domain where the pressure value $\bar{p}$ is assigned. At the generic node on the alignment, only the values $f_{0}, f_{2}, f_{3}, f_{4}, f_{6}$ and $f_{7}$ of the distribution function can be obtained from post-collision values of the previous time step, according to (7). The indeterminacy on $f_{1}, f_{5}$ and $f_{8}$ is exploited to enforce $\bar{p}$, following the first equation in (8):

$$
f_{0}+f_{1}+f_{2}+f_{3}+f_{4}+f_{5}+f_{6}+f_{7}+f_{8}=\frac{\bar{p}}{m c_{\mathrm{s}}^{2}}
$$

However, the above condition does not solve the indeterminacy. As suggested in Ref. [30, the algebraic system can be closed by

$$
\begin{aligned}
& f_{2}-f_{4}+f_{5}+f_{6}-f_{7}-f_{8}=0 \\
& f_{1}-f_{1}^{\text {eq }}=f_{3}-f_{3}^{\text {eq }}
\end{aligned}
$$


According to (8), the first condition in (13) translates the assumption of null component of the fluid velocity parallel to the boundary. The last conditions can be commented as a symmetry assumption on the BGK collision operator (see (7)).

The no-slip condition at external boundaries of the fluid domain were implemented as along the contour of solid particles within the lattice. Its description is therefore postponed to the next subsection.

\section{$2.3 \quad$ Solid-fluid coupling}

For geometrical reasons, the pore network in densely packed two-dimensional granular materials is poorly connected. This leads to unrealistic values of permeability as well as of the drag action on individual particles, compared to real (three-dimensional) granular materials of corresponding grains size distribution. To overcome this limitation, the two-dimensional LB calculation is commonly performed by taking into account a reduction of the particle radii. A reduction factor of 0.8 was assumed for the numerical simulation presented in this work, in line with previous studies on water saturated granular materials [7, 16, 17]. The so-obtained 'hydraulic radius' was used to locate the solid-fluid boundary for the generic solid particle within the LB lattice, as illustrated in Fig. 2

The no-slip condition along fixed as well as moving solid-fluid boundaries was imposed following the procedure proposed in Ref. [31, which consists in a smoothening of the original 'bounce-back rule' 32 by spatial interpolation. The procedure can be illustrated for the lattice node $F$ and velocity $\mathbf{c}_{6}$ in Fig. 2. The relevant value of the distribution function at time $t+\Delta t$ is computed as

$$
\begin{gathered}
f_{6}\left(\mathbf{x}_{F}, t+\Delta t\right)=\overbrace{2 q f_{8}^{\mathrm{c}}\left(\mathbf{x}_{F}, t\right)+(1-2 q) f_{8}^{\mathrm{c}}\left(\mathbf{x}_{R}, t\right)}^{=: f_{8}^{\mathrm{c}}\left(\mathbf{x}_{F^{\prime}}, t\right)} \\
-\frac{2 \omega_{8} \rho}{m c_{\mathrm{s}}^{2}} \mathbf{c}_{8} \cdot \mathbf{v}_{B} \quad \text { if } \quad \mathbf{q}=\frac{\left\|\mathbf{x}_{B}-\mathbf{x}_{F}\right\|}{\left\|\mathbf{x}_{S}-\mathbf{x}_{F}\right\|}<\frac{1}{2}
\end{gathered}
$$

in which the position vectors $\mathbf{x}_{B}, \mathbf{x}_{F}, \mathbf{x}_{F^{\prime}}$ and $\mathbf{x}_{S}$ refer to the respective nodes. The post-collision value at the fictitious node $F^{\prime}$ is computed by linear interpolation based on the corresponding values at the actual lattice nodes $R$ and $F$. The fictitious node is located at the starting position of the path $F^{\prime} B F$ that a fluid particle travelling with the relevant lattice velocities should run to reach $F$ in $\Delta t$. The path includes the collision with the solid boundary at $B$. The second term on the right hand side of (14) takes into account the effect of velocity $\mathbf{v}_{B}$ of the solid particle at the same position. The case when $q \geq 1 / 2$ is treated with a similar interpolation 31 .
The motion of solid particles causes lattice nodes to be progressively subtracted from or added to the fluid domain. At the former nodes, the values of the distribution function were simply let undetermined. The latter nodes required a more elaborated treatment. Assume for example that node $F$ in Fig. 2 is occupied by the solid particle at time $t$ and then added back to the fluid domain at $t+\Delta t$. Then the post-collision values $f_{\alpha}^{\mathrm{c}}\left(\mathbf{x}_{F}, t\right)$ are undetermined. A criterion is then required to define replacement values in order to compute e.g. (7), for $\mathbf{x}=\mathbf{x}_{R}$, and (14). In this study we followed the criterion proposed in Ref. 33. Namely, the missing post-collision values were replaced by equilibrium ones obtained from (9) by assigning meaningful values of $p$ and $\mathbf{u}$ based on the information at time $t$. In particular, $p$ was computed by average over the neighbouring nodes in the fluid domain and $\mathbf{u}$ was replaced by the velocity of the solid particle at $\mathbf{x}_{F}$. The criterion requires that $F$ and $R$ are not added to the fluid domain at the same time step, which is granted by the low Mach number assumption. The above treatment of the motion of solid particles within the lattice is however not implicitly mass conservative, which required checks on this feature throughout the whole computation.

The resultant force exerted by the fluid on a given particle were assembled over the contributions arising at each boundary node. Such contributions were computed based on the momentum transferred per unit time by the interacting node of the fluid domain 32. Namely, the contribution at $B$ in Fig. 2 would be computed as

$$
\mathbf{f}\left(\mathbf{x}_{B}\right)=\frac{m h^{2}}{\Delta t}\left(f_{8}^{\mathrm{c}}\left(\mathbf{x}_{F}, t\right) \mathbf{c}_{8}-f_{6}\left(\mathbf{x}_{F}, t+\Delta t\right) \mathbf{c}_{6}\right)
$$

By the analogous procedures were assembled the resultant force moments.

\section{Backward erosion experiment}

Fig. 3 illustrates the boundary conditions and initial configuration of the coupled erosion test. The figure refers to the DE and the LB models, separately. The granular specimen in Fig. 3a was obtained by the 'dry' preparation procedure presented below, in Sect. 3.1. It consists of about 5400 circular particles with radii randomly dispersed between 0.75 and $1.5 \mathrm{~mm}$, and a mass density of $2.65 \mathrm{Mg} \mathrm{m}^{-2}$. On the 'hydraulic' configuration of the same specimen, in Fig. $3 \mathrm{~b}$, the same particles appear with their radii reduced by a factor of 0.8 , as discussed in Sect.2.3. As to the fluid phase, we assumed a mass density $\rho_{\mathrm{f}}$ of $1 \mathrm{Mg} \mathrm{m}^{-2}$ and a dynamic viscosity $\eta$ of $1 \mathrm{mN} \mathrm{s} \mathrm{m}^{-1}$, in reference to usual values for pore water in soil mechanics applications.

During the test, the fluid motion was triggered and sustained by the imposed pressure unbalance between 
the left and right boundaries in Fig. 3b. The unbalance was applied abruptly (within $0.06 \mathrm{~s}$ ), in the form of opposite increments of the inlet and outlet pressure values, and was kept constant until the end of test, after about $16 \mathrm{~s}$. The estimate $\left(p_{\text {in }}-p_{\text {out }}\right) /\left(\rho_{\mathrm{f}} g L\right) \approx 18 \%$, with $g$ the standard gravity and $\mathrm{L}=24 \mathrm{~cm}$ as in Fig. 3p, provides the reference value of hydraulic gradient. The numerical implementation of the above testing condition is described in Sect. 3.2 .

As in our previous, prototype test [13, the whole set of boundary conditions is a conjecture on the physical situation occurring in the region of the propagating front of the erosion pipe. However, in the test presented herein we exploited a more representative specimen. Consistently, we allowed a more general kinematics in its downstream region and reduced the population of fixed particles to a minimal set which did not implicitly favour arching of force chains (see Fig. 3a, cf. Fig. 8a in [13]). Due to the larger linear dimensions of the specimen, also compared to the size of the aperture, the test discussed herein enabled to observe not only the inception but also the propagation of backward erosion. Our observation and analysis of the test are reported in the final subsection.

\subsection{Specimen preparation}

The initial configuration in Fig. 3 was obtained by a two-stage numerical procedure which did not involve solid-fluid coupling.

The first stage of the procedure consisted in a 'lubricated' compaction from an initial arrangement of the particles on a Cartesian grid (allowing free path lengths of the order of the average particle diameter), with randomly assigned radii and linear velocities. During compaction, four rigid confining walls, pairwise parallel to the main grid directions, were moved towards the centre of the specimen. Their velocities were controlled so to ensure quasi-static conditions and obtain the shape factor of the rectangular configuration in Fig. 3. The wall motions were stopped after an average confining pressure of about $35 \mathrm{kN} \mathrm{m}^{-1}$ had been reached and stabilised on each side of the specimen. The simulation was kept running further on, for the numerical time required for the specimen to approach static equilibrium (at both, the macroscopic and the microscopic levels). The contact parameters for the compaction procedure are listed in the relevant column of Table 1 ; neither particle-toparticle nor particle-to-wall bonds were used, and lubricated conditions were enforced by assigning null values of the friction coefficients. The resulting specimen was homogeneous and isotropic, as can be evinced from the granular configuration and normal contact force distribution in Fig. 4 a.

Prior to the second stage of the preparation proce- dure, the 10381 compressive contacts in Fig. 4a were turned into as many bonds, which were initialised as reported in the relevant columns of Table 1 the non-null values of the adhesion, cohesion, and friction parameters at grain-to-grain contacts are representative of a weakly cemented granular soil (with macroscopic cohesion of a few $10 \mathrm{kN} \mathrm{m}^{-1}$ ). The same values were assigned to grainto-wall contacts at the left, top and bottom rigid walls that confined the granular configuration in Fig. 4a. In contrast, no bond was activated between the right confining wall and the particles in contact.

The second stage of the preparation procedure was meant to simulate the effect of localised unloading, prior to erosion, in the region ahead of the pipe front. During this stage, the right confining wall was gently removed, while preventing the motion of the black-coloured particles in Fig. 3a. The relevant effect in terms of stress transmission through force chains can be understood by comparing Fig. 4a (end of isotropic compaction) to Fig. $4 \mathrm{~b}-\mathrm{d}$ (after full removal of the right wall). The contact network in Fig. 4 b, comprising both compressive unilateral contacts and bonds, appears as almost entirely unloaded: most normal force values are negligible if compared to Fig. 4 a, except in the region opposite to the removed wall. The same network is split into bonds and unilateral contacts in Figs. 4. and 4 $\mathrm{d}$, respectively. The bond network in Fig. 4c is characterised by a significant number of shortly-connected paths and isolated bonds. This is the result of a process of diffuse damage whose emphasis on the right side of the specimen is due to more pronounced unloading and deformation. As can be observed in Fig. 4 $\mathrm{d}$, bond breakage leaves room for self-organised compressive force chains, with a clear arching effect in response to unloading on the right side of the specimen. In Fig. $4 \mathrm{~b}$-d, the very few particles which overstepped the original right boundary of the specimen (during the wall removal procedure) have been removed to facilitate the analysis of the erosion test in the next subsection.

\subsection{Numerical implementation of the coupled test}

The domain in Fig. 3b was discretised on a D2Q9 lattice with characteristic length $h$ of about $25 \mu \mathrm{m}$, for a total number of $55,877,854$ nodes. The time step interval $\Delta t$ of the LB algorithm was set as $6 \mu \mathrm{s}$, namely 20 times larger than the one of the DE algorithm. To fit the aforementioned values of fluid mass density and dynamic viscosity, the relaxation time $\tau$ was set as approximately $0.529 \Delta t$, based on 11 .

The pressure-velocity and no-slip boundary condition in Fig. 3b were implemented as described in Sects. 2.2 and 2.3 respectively. Additionally, an original regularisation procedure was employed that allowed the solid 

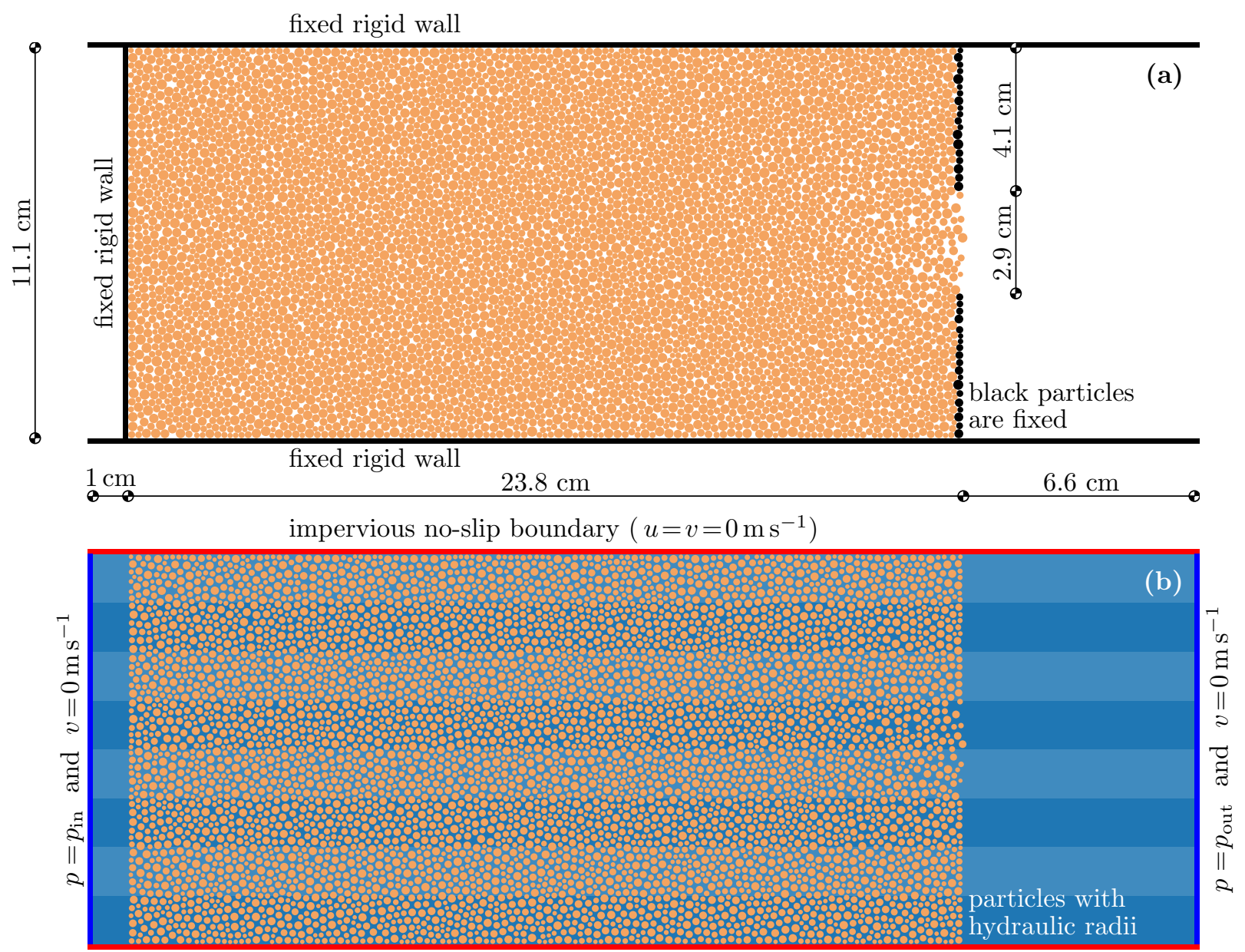

impervious no-slip boundary $\left(u=v=0 \mathrm{~m} \mathrm{~s}^{-1}\right)$

Figure 3: Coupled simulation: overall geometry, initial granular configuration and boundary conditions of the DE (a) and LB (b) models. The macroscopic fluid velocity $\mathbf{u}$ is represented in terms of the components $u$ and $v$ in the horizontal and vertical directions of the figure, respectively. The domain decomposition in 8 sectors for the parallel execution of the LB algorithm is shown in (b) by alternate shades of the background colour.

\begin{tabular}{|c|c|c|c|c|c|}
\hline & \multirow{3}{*}{$\begin{array}{c}\text { Compaction } \\
\text { procedure }\end{array}$} & \multicolumn{3}{|c|}{ Initialisation of the bonds } & \multirow{3}{*}{$\begin{array}{c}\text { After failure } \\
\text { of a bond }\end{array}$} \\
\hline & & \multirow{2}{*}{ Grain-to-grain } & \multicolumn{2}{|c|}{ Grain-to-wall } & \\
\hline & & & Right wall & Other walls & \\
\hline$k_{\mathrm{n}}\left[\mathrm{Nm}^{-1}\right]$ & $5.4 \cdot 10^{7}$ & $5.4 \cdot 10^{7}$ & $5.4 \cdot 10^{7}$ & $5.4 \cdot 10^{7}$ & $5.4 \cdot 10^{7}$ \\
\hline$k_{\mathrm{t}}\left[\mathrm{N} \mathrm{m}^{-1}\right]$ & $5.4 \cdot 10^{7}$ & $5.4 \cdot 10^{7}$ & $5.4 \cdot 10^{7}$ & $5.4 \cdot 10^{7}$ & $5.4 \cdot 10^{7}$ \\
\hline$c_{\mathrm{n}}\left[\mathrm{Ns} \mathrm{m}^{-1}\right]$ & 500 & 500 & 500 & 500 & 500 \\
\hline$c_{\mathrm{t}}\left[\mathrm{Ns} \mathrm{m}^{-1}\right]$ & 500 & 500 & 500 & 500 & 500 \\
\hline$A \quad[\mathrm{~N}]$ & 0 & 40 & 0 & 40 & 0 \\
\hline$C \quad[\mathrm{~N}]$ & 0 & 80 & 0 & 80 & 0 \\
\hline$\mu$ & 0 & 0.55 & 0.55 & 0.55 & 0.55 \\
\hline
\end{tabular}

Table 1: DE model: constitutive parameters used during the compaction procedure and after intialisation or failure of the bonds. 

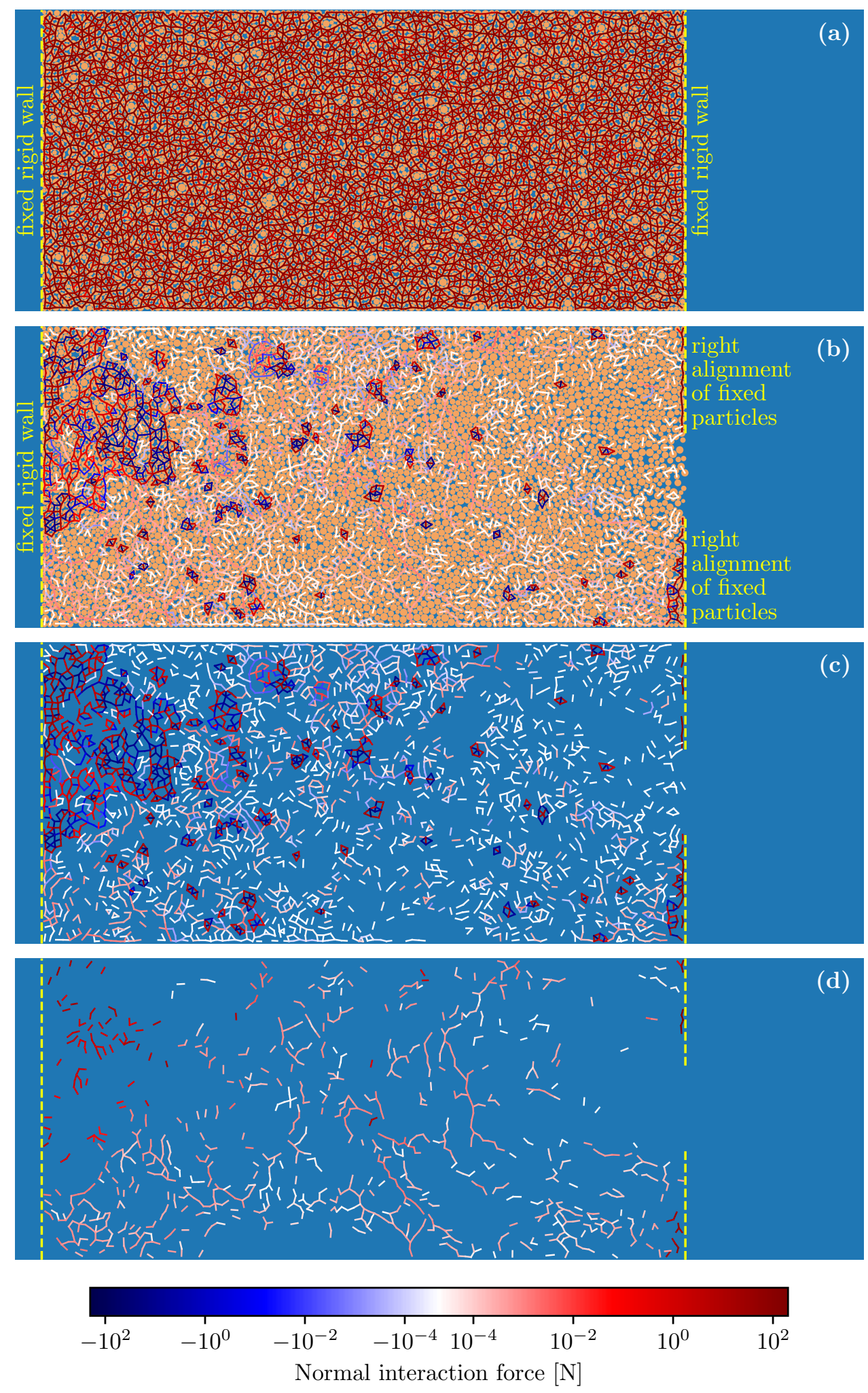

Figure 4: Specimen preparation procedure: granular configuration and force chain network at the end of isotropic compaction (a) and after removal of the right confining wall (b-d). Separate contributions of bonds and unilateral contacts in (b) are reported in (c) and (d), respectively. Yellow dashed lines mark the right alignment of the fixed particles in Fig. 3 a. 
particles to exit the LB domain through the right, outlet boundary (cf. [7, 13]). Due to a number of technicalities, the description of this aspect is however postponed to a further publication.

The LB kernel of the in-house code was designed for parallel execution, by domain decomposition, on shared memory architectures. The simulation was executed by a processor of type Intel ${ }^{\circledR}$ Xeon ${ }^{\circledR}$ E5-2690, with 8 cores and $2.9 \mathrm{GHz}$ clock speed. The corresponding decomposition of the LB domain in 8 sectors of equal size is shown in Fig. 3p. The total computing time was about 30 days.

\section{$3.3 \quad$ Results}

Figs. 5 and 6 illustrate the mobilisation and flow of particles during the initial and final stages of the coupled erosion test, respectively. In both figures, the populations of 'quasi-static' (blue coloured) particles and of 'fluidised' (red coloured) particles are detected based on the norm of their linear velocity being smaller than $0.004 \mathrm{~m} \mathrm{~s}^{-1}$ or larger than $0.02 \mathrm{~m} \mathrm{~s}^{-1}$, respectively. Particles having exited the right fluid boundary are also considered as part of the fluidised mass. In the first stage of the experiment, illustrated in Fig. 5, an evolving region of mobilised (yet not fluidised) particles first appears on the downstream side of the specimen, close to the unconfined sector between the two vertical alignments of fixed particles (dashed lines). The left limit of the mobilised region is inferred visually and marked by a vertical dotted line, in each plot the of the figure. This observation suggests a nearly constant backward propagation celerity, whose value was estimated as about $0.0836 \mathrm{~m} \mathrm{~s}^{-1}$ by linear regression. Towards the end of the numerical experiment and of the erosion process, illustrated in Fig. 6. the fluid flow progressively drags the mobilised particles out of the specimen, and the erosion pipe progressively appears.

The fluidisation threshold $\left(0.02 \mathrm{~m} \mathrm{~s}^{-1}\right)$ is by definition a lower bound for the linear velocities of particles in motion with the fluid. Its value was therefore chosen as representative of the localised fluid flow induced by the erosion process itself, as illustrated in the maps of fluid velocity norm in Fig. 7. Namely, the selected threshold value delineates both (i) the fluid stream out of the central, unconstrained and higher-porosity granular region in the first stage of the test (Fig. 7a, cf. Fig. 5d) and (ii) the profile of the erosion pipe in the final stage of the test (Fig. 7b, cf. Fig. 6d), with significant precision. According to this definition, in the initial stage of the erosion process (Fig. 5), the mobilised granular mass is fluidised in the right free-flow region, out of the specimen, while in the final stage the fluidisation occurs within the erosion pipe (Fig. 6, except for Fig. 6d as discussed below).
The quasi-static threshold $\left(0.004 \mathrm{~m} \mathrm{~s}^{-1}\right)$ was identified as a compromise between several criteria, among which that it be as small as possible, yet beyond the range of 'measurement noise'. In this sense, and taking fluidisation as the reference process, the candidate order of magnitude was identified of $10^{-3} \mathrm{~m} \mathrm{~s}^{-1}$, namely one order higher than the variance of the linear velocities in the fluidised granular mass (estimated within the observation window in Fig. 6). The threshold value was then determined with more accuracy based on the backward propagation of the mobilised regions in Fig. 5. We mention in particular that the same linear regression procedure by which we computed the propagation celerity also provides an estimate of the initial (supposedly null) horizontal extension of the mobilised mass, depending on the candidate quasi-static threshold. For the selected threshold value, the estimated initial extension was satisfactorily as small as roughly $1 \mathrm{~cm}$. Such estimate was only negligibly improved by attempting slightly higher quasi-static velocity thresholds, which resulted in an undesirable degradation of the contours of the mobilised granular regions (compared to Fig. 5).

The charts in Fig. 8 illustrate the evolution of the mass fractions of quasi-static particles (red squares) and of fluidised particles (blue circles) during the coupled simulation. The time instants on the left and right sides of the top axis correspond to the sequences of plots in Figs. 5 and 6, respectively. From the evolution of the fluidised mass fraction, it can be inferred that, except for the final stage, the process is characterised by a practically constant mass-erosion rate. After roughly the first half of the simulation $(t>7 \mathrm{~s})$, the mobilised mass is not anymore sourcing from the quasi-static one, whose fraction keeps stationary. Towards the end of the test $(t>16 \mathrm{~s})$, the populations of quasi-static and fluidised particles become complementary (i.e. sum up to $95 \%$ of the initial mass of the specimen), which marks the end of the erosion process. The aforementioned constant erosion rate is lost around $t=11 \mathrm{~s}$, due to an unstable behaviour that precedes the clogging event centred at $t=14.52 \mathrm{~s}$ and apparent in Fig. 10.

The statistics in Table 2 show that almost all damage, in terms of bond breakage, occurs during the unloading stage of the preparation procedure. Namely, about $63 \%$ of the initial bond population is lost during removal of the right confining wall, resulting in 525 separated networks of bonded particles (shortly 'bond networks') being generated from the initial, single one. At least half of the population of new bond networks generated during the wall removal procedure consists of minimal individuals (i.e. of networks with only one bond, see Fig. 4k) as can be inferred from the relevant median values of the number of particles, number of bonds and coordination number over the network population. Only about $9 \%$ of additional losses of bonds occur during the erosion 

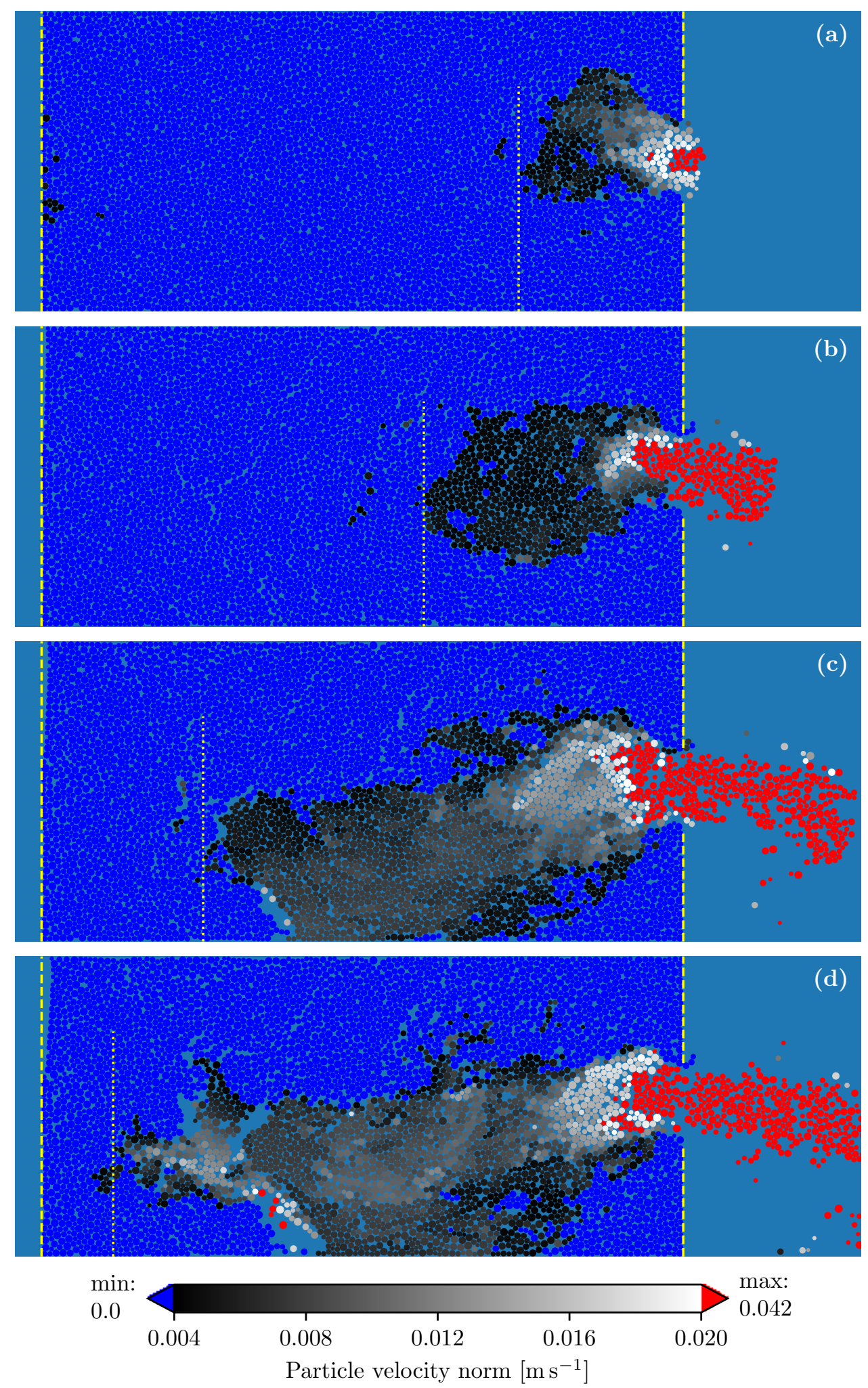

Figure 5: Coupled simulation: linear velocity norm of particles at $\mathrm{t}=0.6 \mathrm{~s}(\mathrm{a}), 1.8 \mathrm{~s}(\mathrm{~b}), 3.0 \mathrm{~s}$ (c) and $4.2 \mathrm{~s}(\mathrm{~d})$. Quasi-stationary particles (norm $<0.004 \mathrm{~m} \mathrm{~s}^{-1}$ ) and fluidised particles (norm $>0.02 \mathrm{~m} \mathrm{~s}^{-1}$ ) are plotted in blue and red colours, respectively. Yellow dashed lines are defined in Fig. $4 \mathrm{~b}$. The horizontal extension of the mobilised regions are marked by yellow dotted lines. 

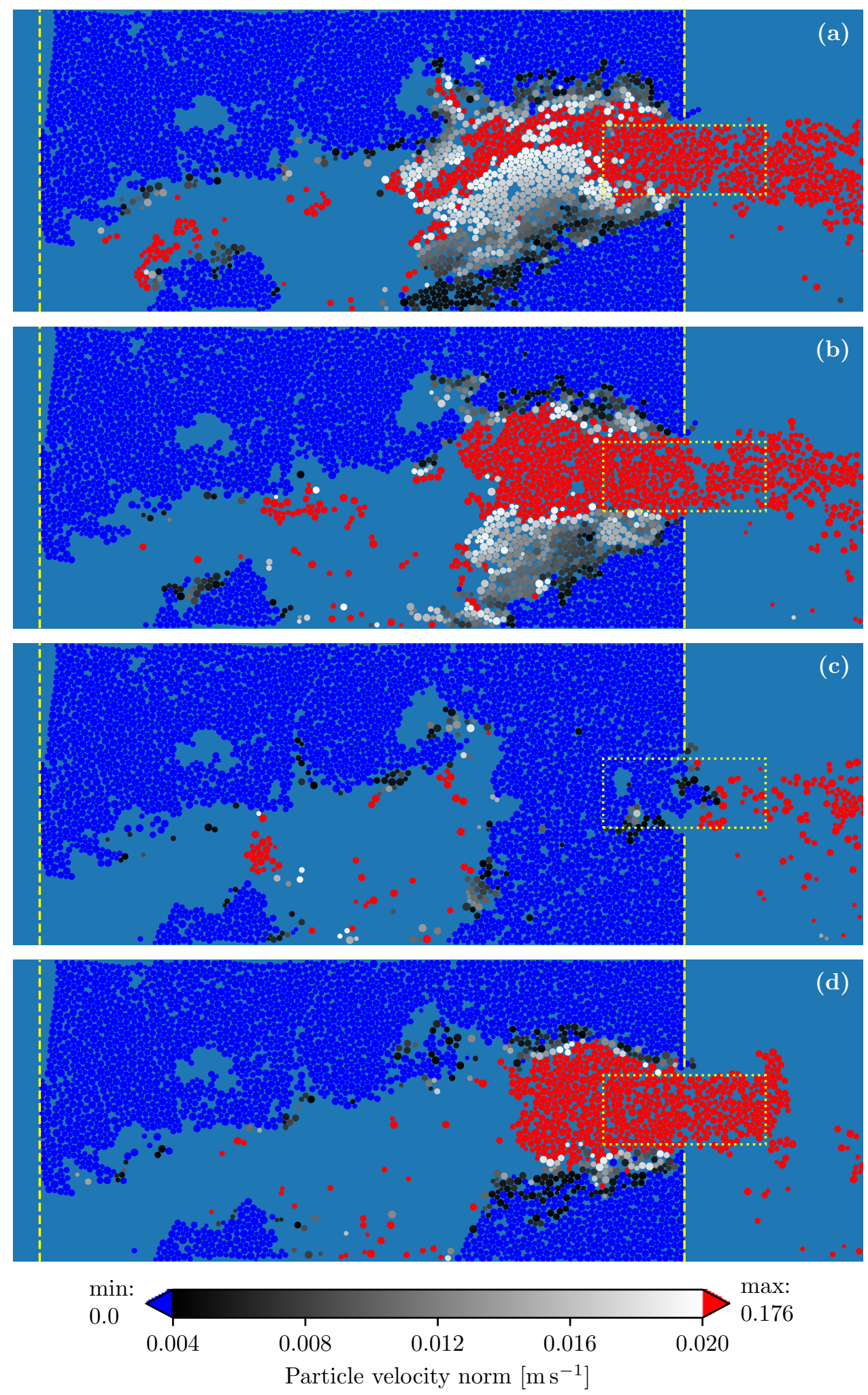

Figure 6: Coupled simulation: linear velocity norm of particles at $\mathrm{t}=12.12 \mathrm{~s}(\mathrm{a}), 13.32 \mathrm{~s}(\mathrm{~b}), 14.52 \mathrm{~s}(\mathrm{c})$ and 15.72 $\mathrm{s}(\mathrm{d})$. Quasi-stationary particles (norm $<0.004 \mathrm{~m} \mathrm{~s}^{-1}$ ) and fully-eroded particles (norm $>0.02 \mathrm{~m} \mathrm{~s}^{-1}$ ) are plotted in blue and red colours, respectively. Yellow dashed lines are defined in Fig. 4 $4 \mathrm{p}$. The fixed yellow dotted rectangle is an observation window referred to in the text. 

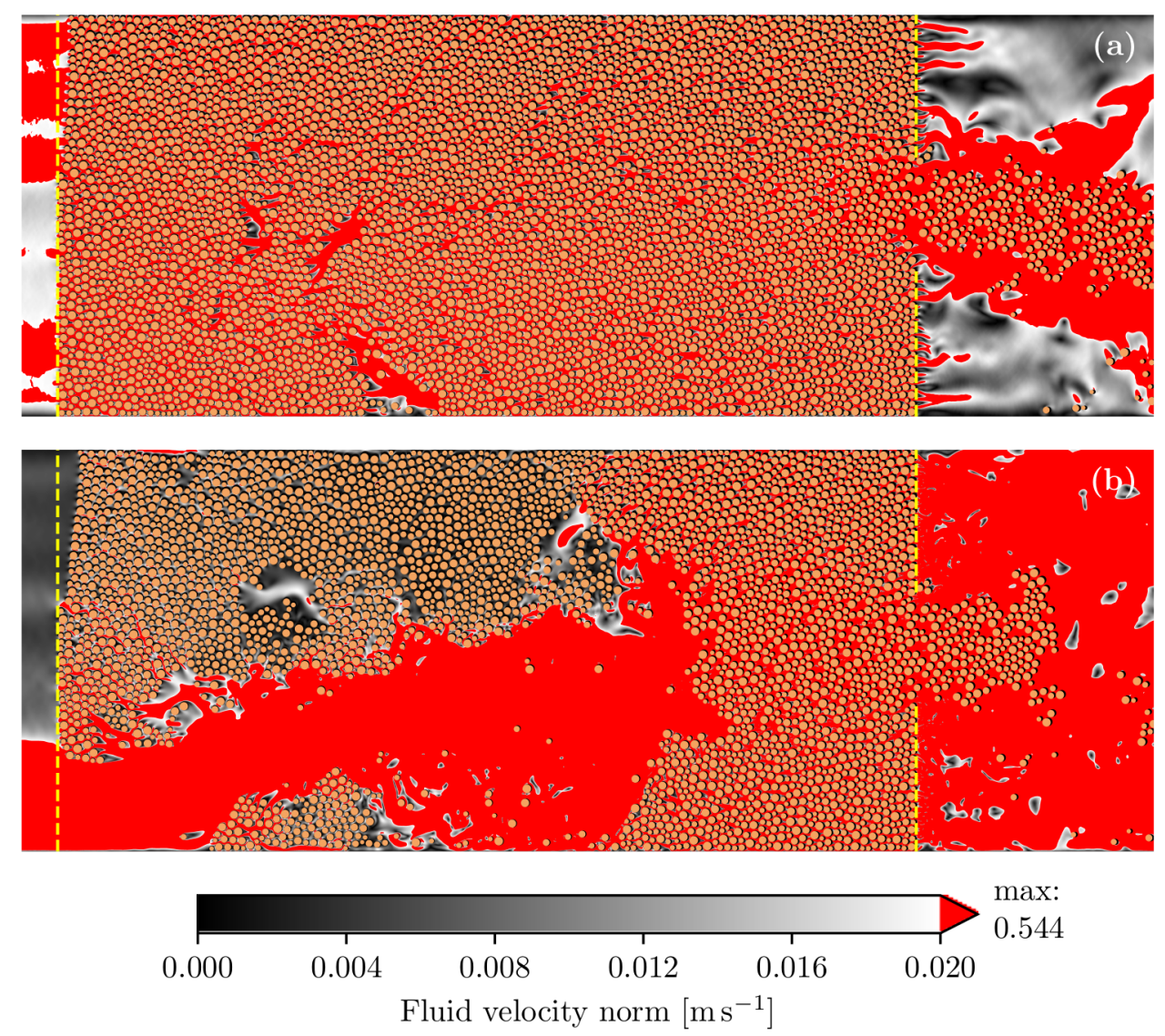

Figure 7: Coupled simulation: fluid velocity norm at $t=4.2 \mathrm{~s}$ (a, see Fig. 5d) and at $t=15.72 \mathrm{~s}$ (b, see Fig. 6 $\mathrm{d}$ ). Values above the fluidisation threshold $\left(\right.$ norm $>0.02 \mathrm{~m} \mathrm{~s}^{-1}$ ) are represented in red colour. Yellow dashed lines are defined in Fig. 4p.

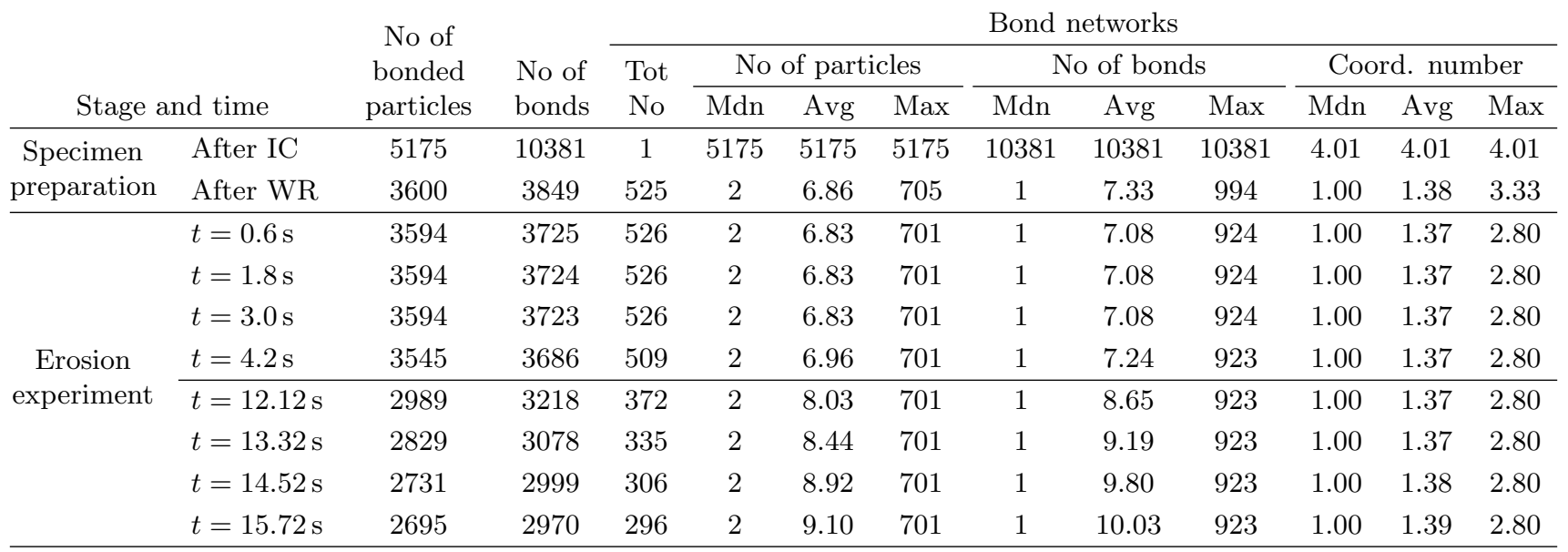

Table 2: Bond degradation during the preparation procedure (IC: isotropic compression; WR: wall removal) and the coupled simulation (same time instants as in Fig. 5 and 6). The evolution of bond networks is characterised in terms of their total number and of the median, average and maximum values of the per-network number of particles, number of bonds and coordination number. 


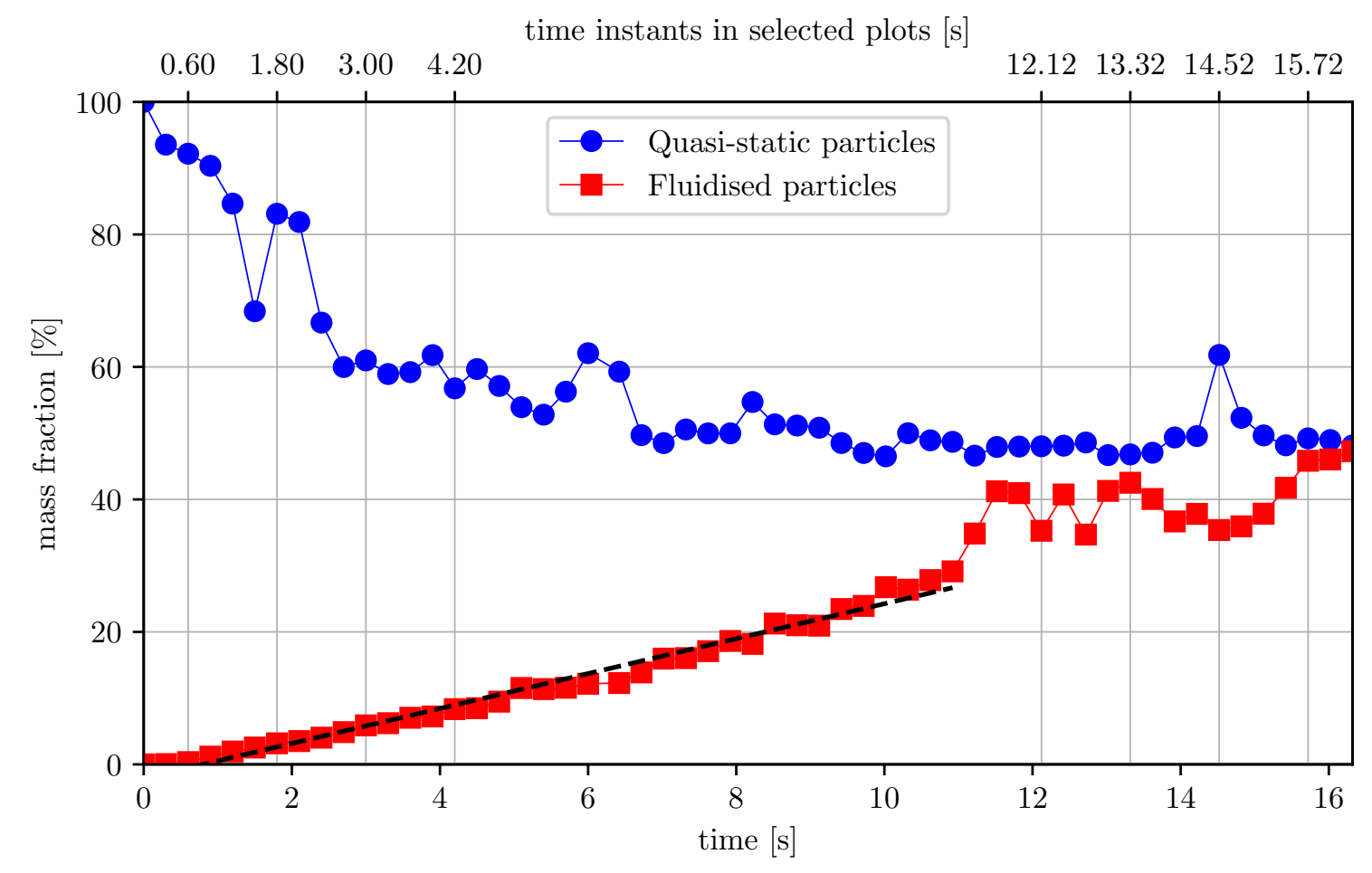

Figure 8: Coupled simulation: time evolution of the masses of the quasi-stationary and fluidised particles, as fractions of the initial mass of the specimen (see Figs. 5 and 6, which refer to the time instants reported on the top axis of this figure). The initial mass erosion rate is pointed out by a dashed line.

experiment, despite a substantial reduction of the number of bond networks. This is due to the comminution of small networks, as can be inferred by the increasing average values of the number of particles and bonds. Conversely, the largest bond networks undergo comparatively no loss of bonds during the erosion experiment, as demonstrated by further available statistics not reported herein. In Fig. 9, the total number of bonds in the network to which each particle belongs, denoted as 'belonging network bond population', is plotted using a two-colour map. The visualisation emphasises how the backward erosion path is largely influenced by the damage which affects the specimen during the unloading procedure described in Sect. 3.1. Indeed, the erosion pipe in Fig. 9 $\mathrm{b}$ is almost entirely located in the magenta region of relatively small bond networks in Fig. 9a.

The internal response of the specimen to the imposed hydraulic loading is illustrated for $t=0.6 \mathrm{~s}$ in Fig. 10 . representative of the initial stage of the test (see also Figs. 5p and 8). Arching through force chains clearly appears as self-organised resistance mechanism (Fig. 10 ab), primarily of unilateral contacts (Fig. 10d) and secondarily of the residual bonds (Fig. 10.). In a more advanced stage, e.g. for $t=13.32 \mathrm{~s}$ in Fig. 11 (see also Figs. 6b and 8), the fluidised mass flows freely within the breach due to the vanishing of arching. However, the clogging of the aperture at $t=14.52 \mathrm{~s}$, inferred from Figs. 6r and 8, is clearly due to the re-onset of arching, shown in Fig. 12. Our previous comments on the selforganisation of arching, and its transient nature, also apply to this stage.

\section{Conclusions}

We presented a numerical experiment for the investigation of backward erosion piping at the grain scale, in the pipe front region. The study benefited of a previous prototype experiment performed by means of essentially the same numerical technique (the coupling of the DE and LB methods) 13. However, significant advances were enabled in this study by the parallelisation of the LB algorithm. They include significantly higher degrees of resolution of the fluid domain and of representativeness of the granular specimen.

In the model pipe-front region, the progress of erosion was observed from inception to exhaustion. Among the most original contributions of our analysis, we emphasise the definitions of the 'quasi-static', 'mobilised' and 'fluidised' particle populations by means of physically motivated identification of two velocity thresholds. These definitions enabled to observe a constant erosion 

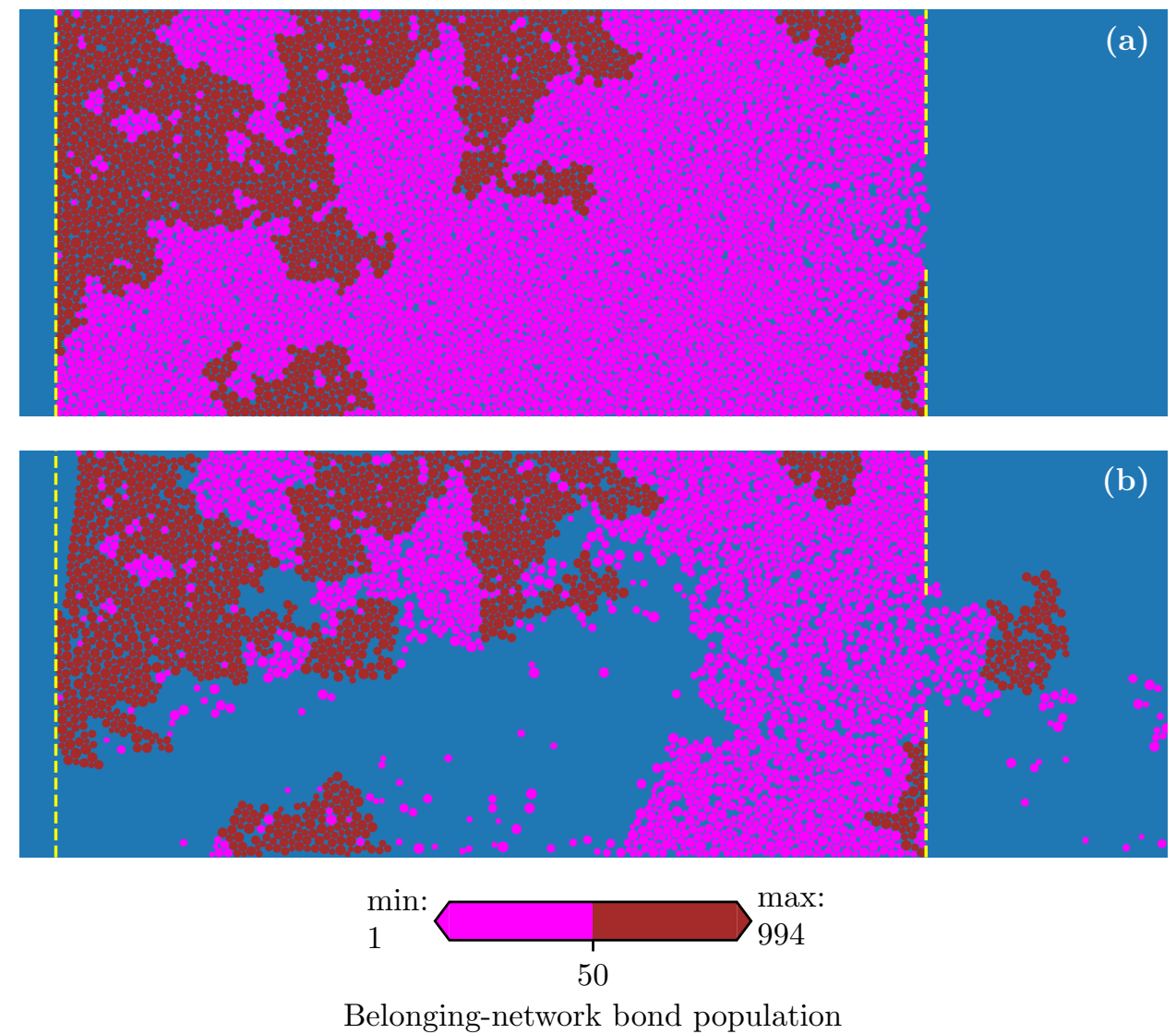

Figure 9: Belonging-network bond population (i.e. the total number of bonds in the network to which the particle belongs) after removal of the right confining wall (a) and towards the end of the coupled simulation (b, at $t=15.72 \mathrm{~s})$. Yellow dashed lines are defined in Fig. 4 b.

rate in the initial stage of the numerical experiment, an almost constant celerity of backward propagation for the region of mobilised particles, and a clogging event in an advanced stage of the test. As previously suggested [13, arching through force chains appeared to be a main resistance mechanism, promoted by the self-organisation of (primarily) the unilateral contact network under quasistatic conditions (e.g. due to clogging). Our statistics on the degradation of the networks of inter-particle cohesive bonds emphasised how the backward erosion path observed during the test was largely influenced by the specimen preparation procedure.

Further analyses will require numerical simulations on even larger specimens, for the boundary conditions to be applied at a 'safer' distance from the candidate pipe region, i.e. to avoid any spurious influences on the pipe path. The observation of possible erosion patterns, e.g. involving mobilisation, fluidisation and clogging of the granular mass, will require the simulation to span much larger time lapses. All in all, these objectives call for more computational efficiency, possibly through more extensive parallelisation paradigms (e.g. on distributed memory architectures) and the use of GPUs. Despite its obvious limitations, two-dimensional modelling still appears to be a main tool for the investigation of backward erosion piping at the grain scale. It allows realistic computation times and enables effective data visualisation and analysis. Discrete three-dimensional modelling is expected to provide a more exhaustive description of the process, in a midterm perspective.

Acknowledgments Research supported by CNRS GDRI GeoMech, GIS VOR 2012, LTDS 2012 and 2018, PRIN 2010-2011 (2010BFXRHS_004) and DiBT 'Computational modeling of erosion' projects. The research also benefited from several invitations of C. Callari at École centrale de Lyon - LTDS. The last author was supported by one $\mathrm{PhD}$ fellowship funded by University of Rome 'Tor Vergata' and by a VINCI mobility programme for $\mathrm{PhD}$ in co-tutorship (Université FrancoItalienne). 

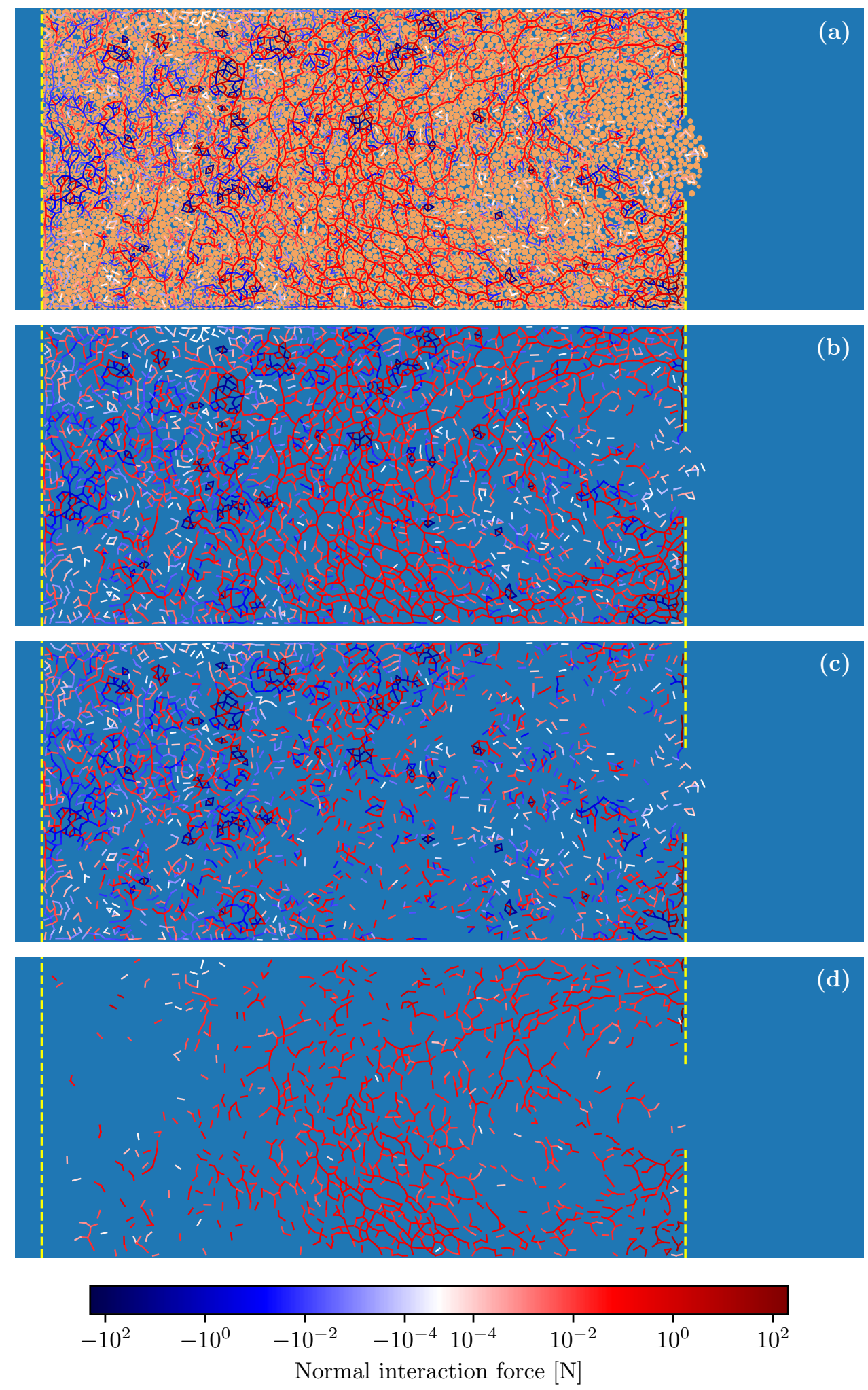

Figure 10: Force chain network at $t=0.6 \mathrm{~s}$, representative of the initial stage of the coupled simulation (see Fig. $5 \mathrm{~b}$ and Fig. 8). Normal interaction forces at bonds and unilateral contacts are plotted, jointly, on the granular configuration (a) and without the granular configuration (b). Separate contributions of bonds and unilateral contacts are plotted in (c) and (d), respectively. Yellow dashed lines are defined in Fig. $4 \mathrm{~b}$. 

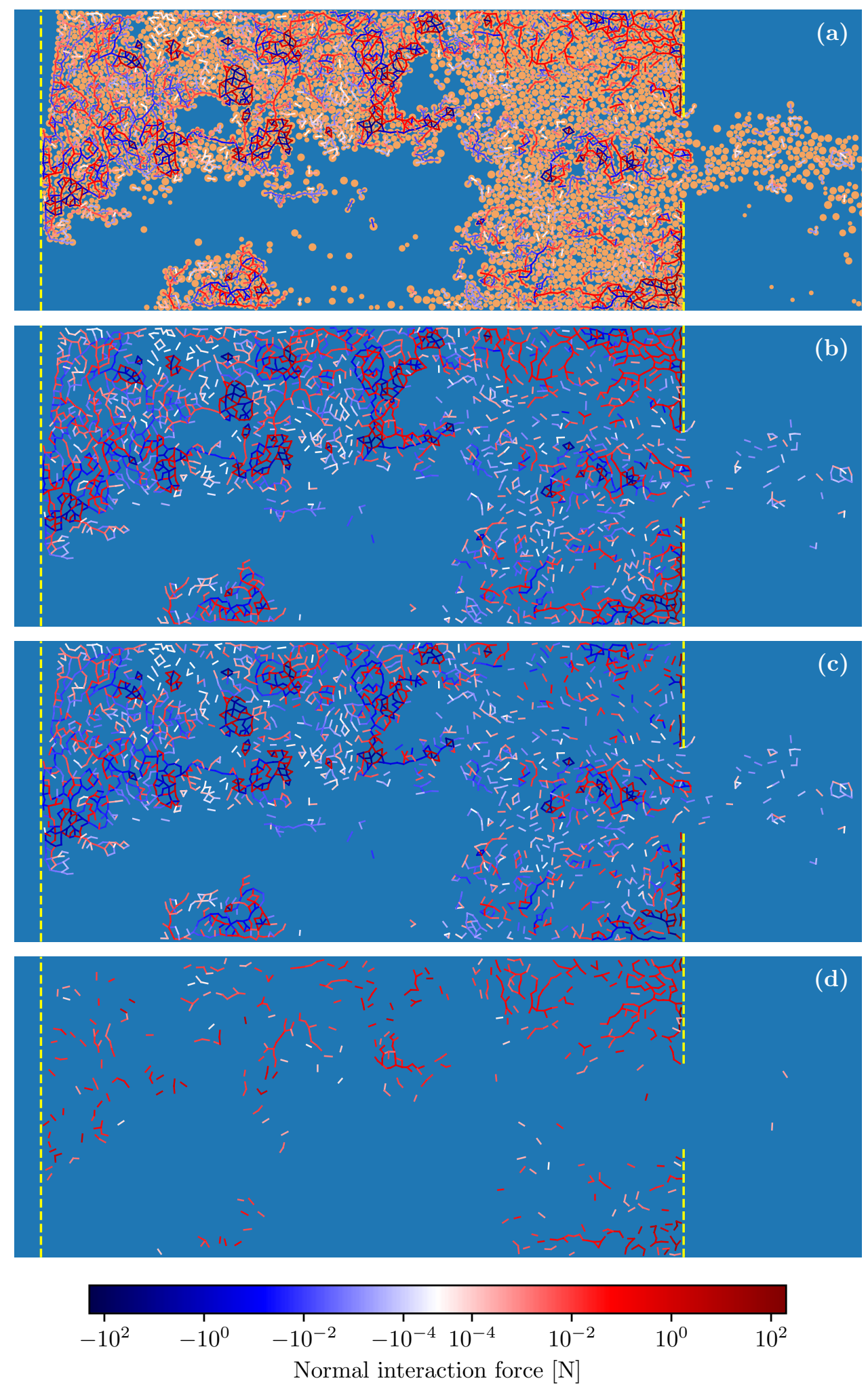

Figure 11: Force chain network at $t=13.32 \mathrm{~s}$, prior to the clogging event (see Fig. 6b and Fig. 8). Normal interaction forces at bonds and unilateral contacts are plotted, jointly, on the granular configuration (a) and without the granular configuration (b). Separate contributions of bonds and unilateral contacts are plotted in (c) and (d), respectively. Yellow dashed lines are defined in Fig. $4 \mathrm{~b}$. 

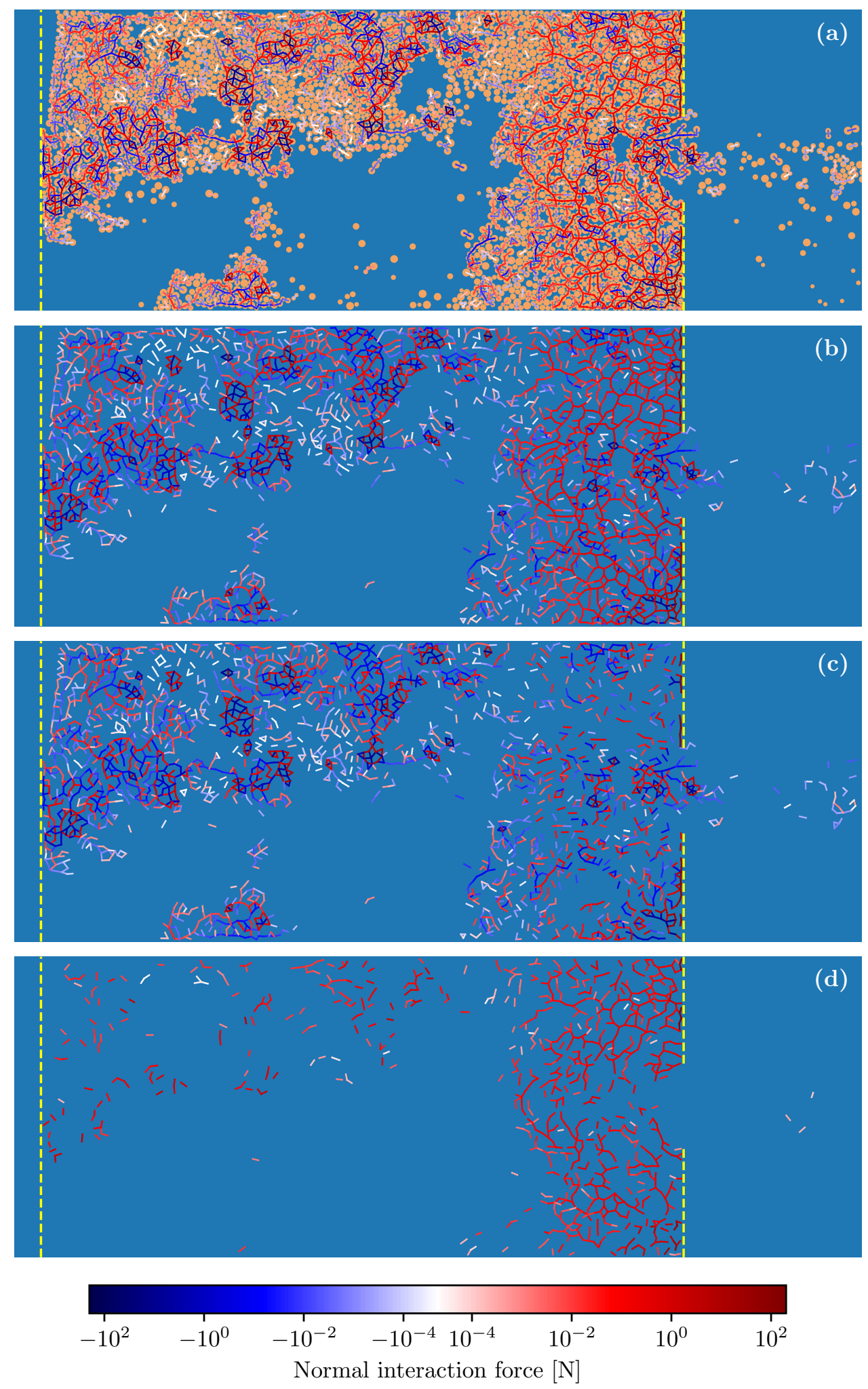

Figure 12: Force chain network at $t=14.52 \mathrm{~s}$, corresponding to the clogging event (see Fig. 6 $\mathrm{d}$ and Fig. 8). Normal interaction forces at bonds and unilateral contacts are plotted, jointly, on the granular configuration (a) and without the granular configuration (b). Separate contributions of bonds and unilateral contacts are plotted in (c) and (d), respectively. Yellow dashed lines are defined in Fig. 4b. 


\section{References}

[1] M. Foster, R. Fell, and M. Spannagle. The statistics of embankement dam failures and accidents. Canadian Geotechnical Journal, 37:1000-1024, 2000.

[2] R. Fell and J.-J. Fry, editors. Internal Erosion of Dams and Their Foundations, Leiden, The Netherlands, 2007. EDF, IREX, Taylor and Francis/Balkema.

[3] S. Bonelli, editor. Erosion of Geomaterials. WileyISTE, 2012.

[4] S. Bonelli, C. Jommi, and D. Sterpi, editors. Internal Erosion in Earthdams, Dikes and Levees. EWGIE: European Working Group on Internal Erosion, Springer International Publishing, 2019.

[5] Chi Fai Wan and Robin Fell. Laboratory tests on the rate of piping erosion of soils in embankment dams. Geotechnical Testing Journal, 27(3):295-303, 2004 .

[6] S. Bonelli and O. Brivois. The scaling law in the hole erosion test with a constant pressure drop. International Journal for Numerical and Analytical Methods in Geomechanics, 32:1573-1595, 2008.

[7] F. Lominé, L. Scholtès, L. Sibille, and P. Poullain. Modeling of fluid-solid interaction in granular media with coupled lattice Boltzmann/discrete element methods: application to piping erosion. International Journal for Numerical and Analytical Methods in Geomechanics, 37(6):577-596, 2013.

[8] A.F. Rotunno, C. Callari, and F. Froiio. Computational modeling of backward erosion piping. In M. Frémond, F. Maceri, and G. Vairo, editors, Models, Simulation, and Experimental Issues in Structural Mechanics, pages 225-234. Springer International Publishing, 2017.

[9] A.F. Rotunno, C. Callari, and F. Froiio. A finite element method for localized erosion in porous media with applications to backward piping in levees. International Journal for Numerical and Analytical Methods in Geomechanics, 43(1):293-316, 2019.

[10] A.F. Rotunno, C. Callari, and F. Froiio. A numerical approach for the analysis of piping erosion in hydraulic works. In Bonelli et al. 4, pages 159-167.

[11] D. Wang, X. Fu, Y. Jie, W. Dong, and D. Hu. Simulation of pipe progression in a levee foundation with coupled seepage and pipe flow domains. Soils and Foundations, 54(5):974-984, 2014.
[12] B. A. Robbins. Numerical modeling of backward erosion piping. In P. Gómez, C. Detournay, R. Hart, and M. Nelson, editors, Applied Numerical Modeling In Geomechanics 2016, Lima, Perú, 7-9 March 2016, pages 551-558. Minneapolis, USA: Itasca International Inc.

[13] D.K. Tran, N. Prime, F. Froiio, C. Callari, and E. Vincens. Numerical modelling of backward front propagation in piping erosion by DEM-LBM coupling. European Journal of Environmental and Civil Engineering, 21(7-8):960-987, 2017.

[14] F. Radjai, S. Nezamabadi, and S. Delenne J.-Y. Luding, editors. Powders and Grains 2017 - 8th International Conference on Micromechanics on Granular Media, volume 140 of EPJ Web of Conferences, Les Ulis, FR, 2017. EDP Sciences.

[15] L. Sibille, F. Lominé, P. Poullain, Y. Sail, and D. Marot. Internal erosion in granular media: direct numerical simulations and energy interpretation. Hydrological Processes, 29(9):2149-2163, 2015.

[16] David F. Boutt, Benjamin K. Cook, Brian J. O. L. McPherson, and J. R. Williams. Direct simulation of fluid-solid mechanics in porous media using the discrete element and lattice-Boltzmann methods. Journal of Geophysical Research: Solid Earth, 112(B10), 2007.

[17] D. F. Boutt, B. K. Cook, and J. R. Williams. A coupled fluidsolid model for problems in geomechanics: Application to sand production. International Journal for Numerical and Analytical Methods in Geomechanics, 35:997, 2011.

[18] P.A. Cundall and O.D.L. Strack. A discrete numerical model for granular assemblies. Gotechnique, 29(1):47-65, 1979.

[19] F. Radjai and F. Dubois, editors. Discrete-element modeling of granular materials. Wiley-ISTE, 2011.

[20] A. A. Mohamad. Lattice Boltzmann Method. Springer London, 2019.

[21] D.O. Potyondy and P.A. Cundall. A bondedparticle model for rock. International Journal of Rock Mechanics and Mining Sciences, 41(8):13291364, 2004.

[22] L. Scholtès and F.-V. Donzé. Modelling progressive failure in fractured rock masses using a $3 \mathrm{~d}$ discrete element method. International Journal of Rock Mechanics and Mining Sciences, 52:18-30, 2012.

[23] L. Scholtès and F.-V. Donzé. A dem model for soft and hard rocks: Role of grain interlocking on 
strength. Journal of the Mechanics and Physics of Solids, 61(2):352-369, 2013.

[24] W.C. Swope, Andersen H.C., Berens P.H, and Wilson K.R. A computer simulation method for the calculation of equilibrium constants for the formation of physical clusters of molecules: Application to small water clusters. The Journal of Chemical Physics, 76(1):637-649, 1982.

[25] P. L. Bhatnagar, E. P. Gross, and M. Krook. A model for collision processes in gases. I. small amplitude processes in charged and neutral onecomponent systems. Physical Review, 94(3):511$525,1954$.

[26] Y.H. Qian and P. d'Humiéres, D.and Lallemand. Lattice BGK models for Navier-Stokes equation. Europhysics Letters, 17(6):479-484, 1992.

[27] A.M. Vasilyev. Introduction to Statistical Physics. Imported Pubn, 1984.

[28] X. He and L.-S Luo. Lattice Boltzmann Model for the incompressible Navier-Stokes equation. Journal of Statistiscal Physics, 88(3-4):927-944, 1997.

[29] D d'Humières. Generalized Lattice Boltzmann equations. In Shizgal D. and Weaver D.P., editors, Rarefied Gas Dynamics: Theory and Simulations. American Institute of Aeronautics and Astronautics, 1992.

[30] Q. Zou and X. He. On pressure and velocity boundary conditions for the lattice Boltzmann BGK model. Physics of Fluids, 9(6):1591-1598, 1997.

[31] M. Bouzidi, M. Firdaouss, and P. Lallemand. Momentum transfer of a Boltzmann-lattice fluid with boundaries. Physics of Fluids, 13(11):3452-3459, 2001.

[32] A.J.C. Ladd. Numerical simulations of particulate suspensions via a discretized Boltzmann equation. Part 1. Theoretical foundation. Journal of Fluid Mechanics, 271:285-309, 1994.

[33] C.K. Aidun, Y. Lu, and E.-J Ding. Direct analysis of particulate suspensions with inertia using the discrete Boltzmann equation. Journal of Fluid Mechanics, 373(1):287-311, 1998. 